2021, Band 9, Heft 1/2

Seiten 213-224

zeitschrift-suburban.de

10.36900/suburban.v9i1/2.696

\title{
Analog Algorithm - Landscapes of Machine Learning
}

Susanne Huth

mit einem Kommentar von Gala Nettelbladt

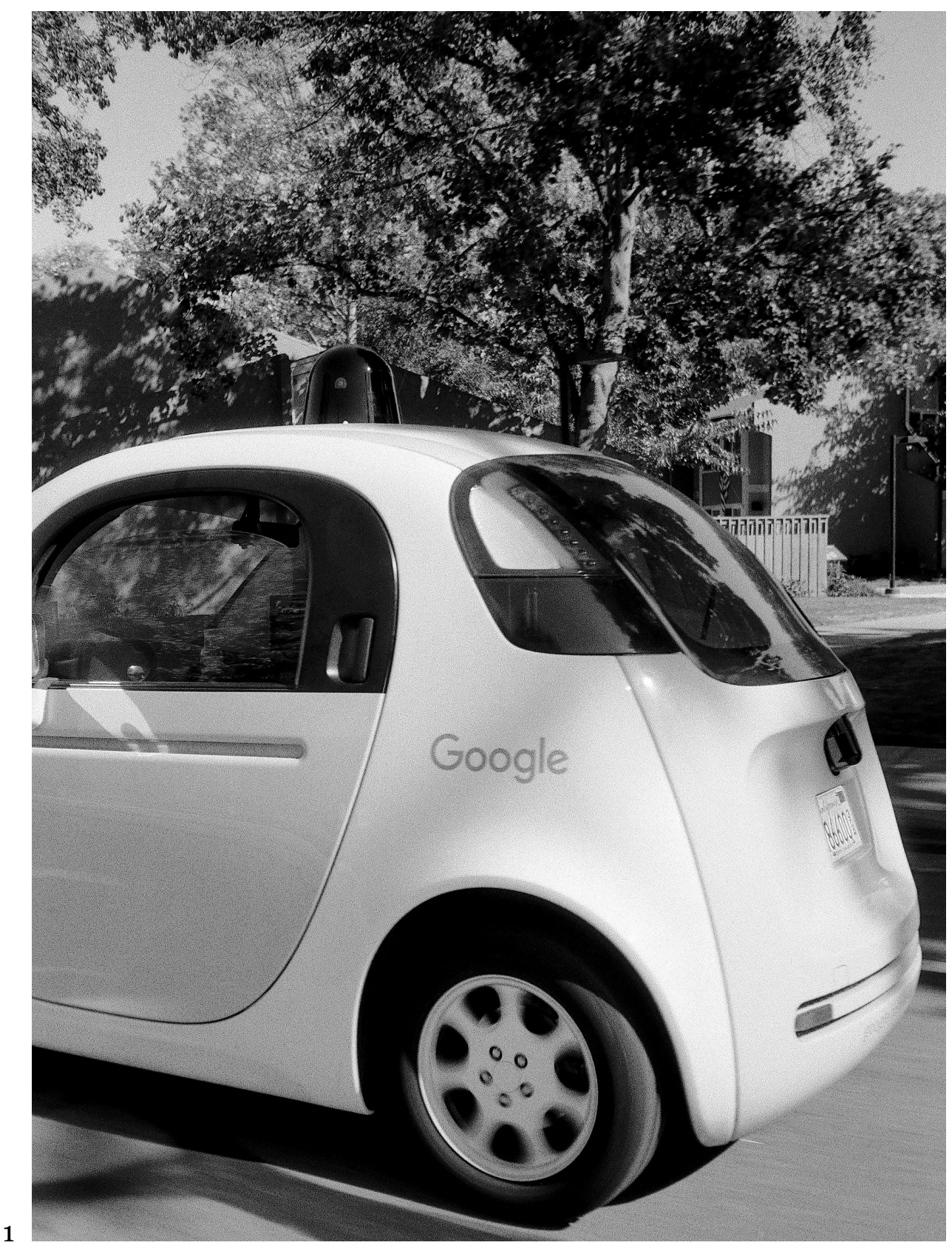




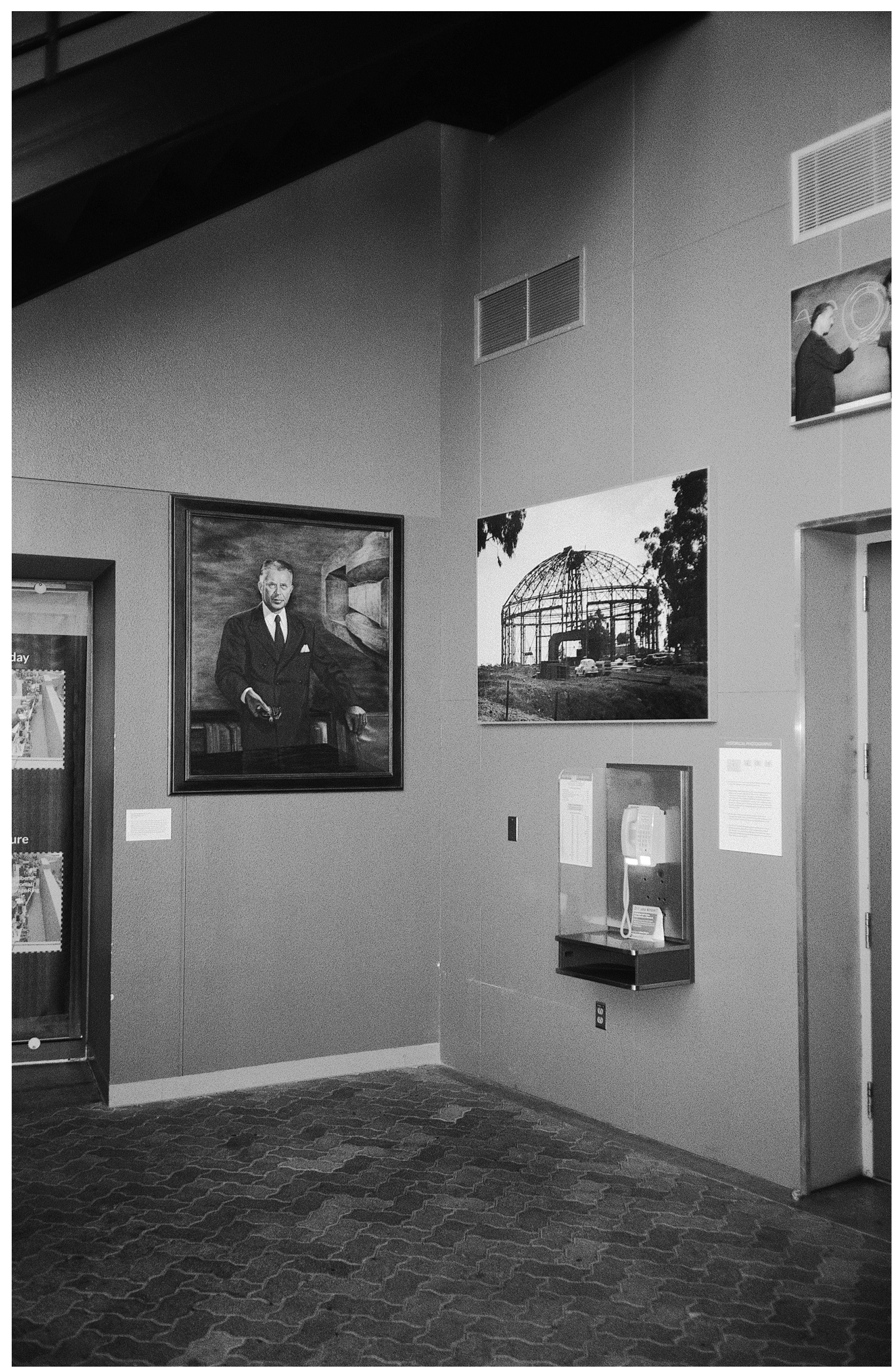




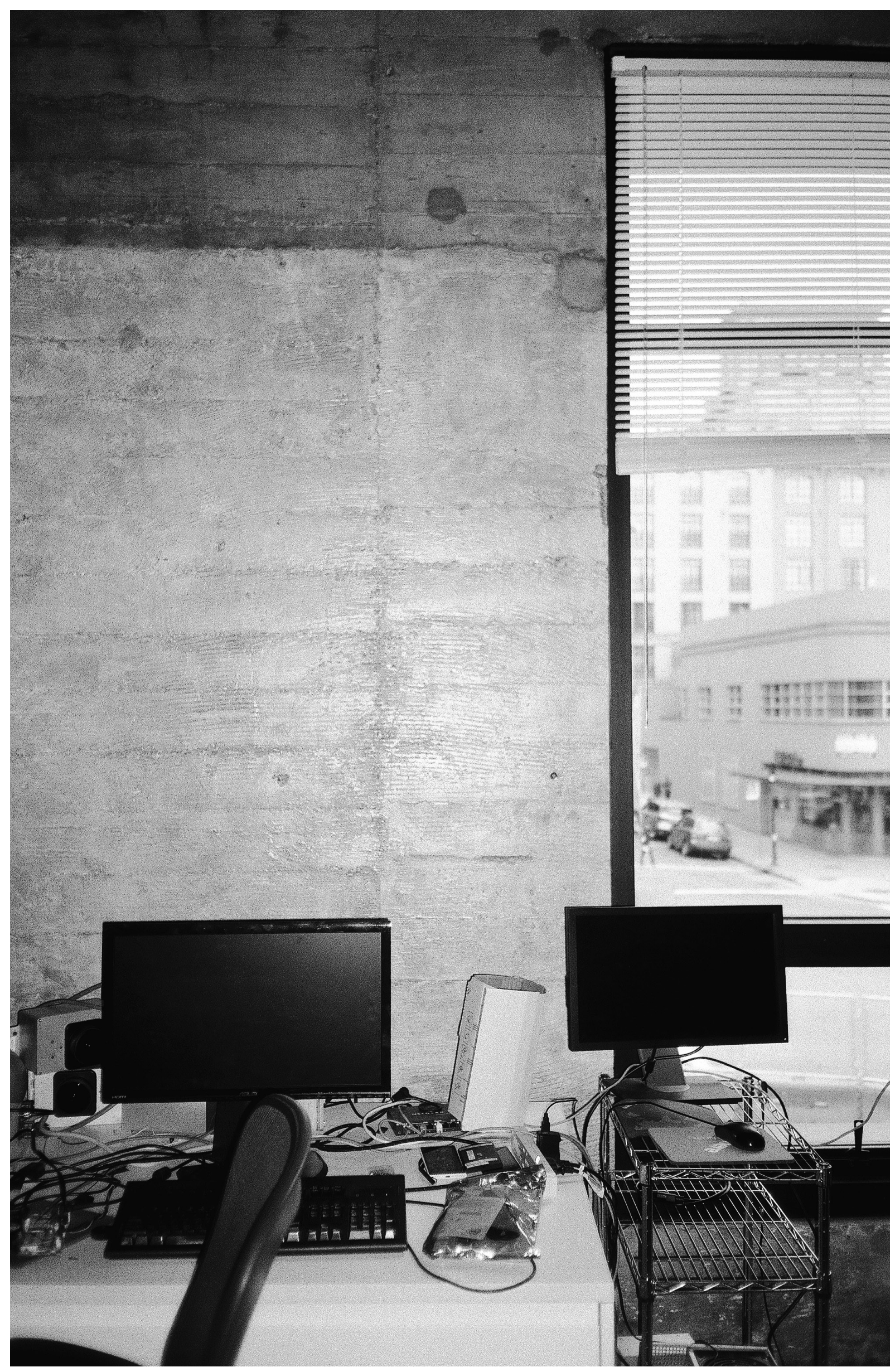




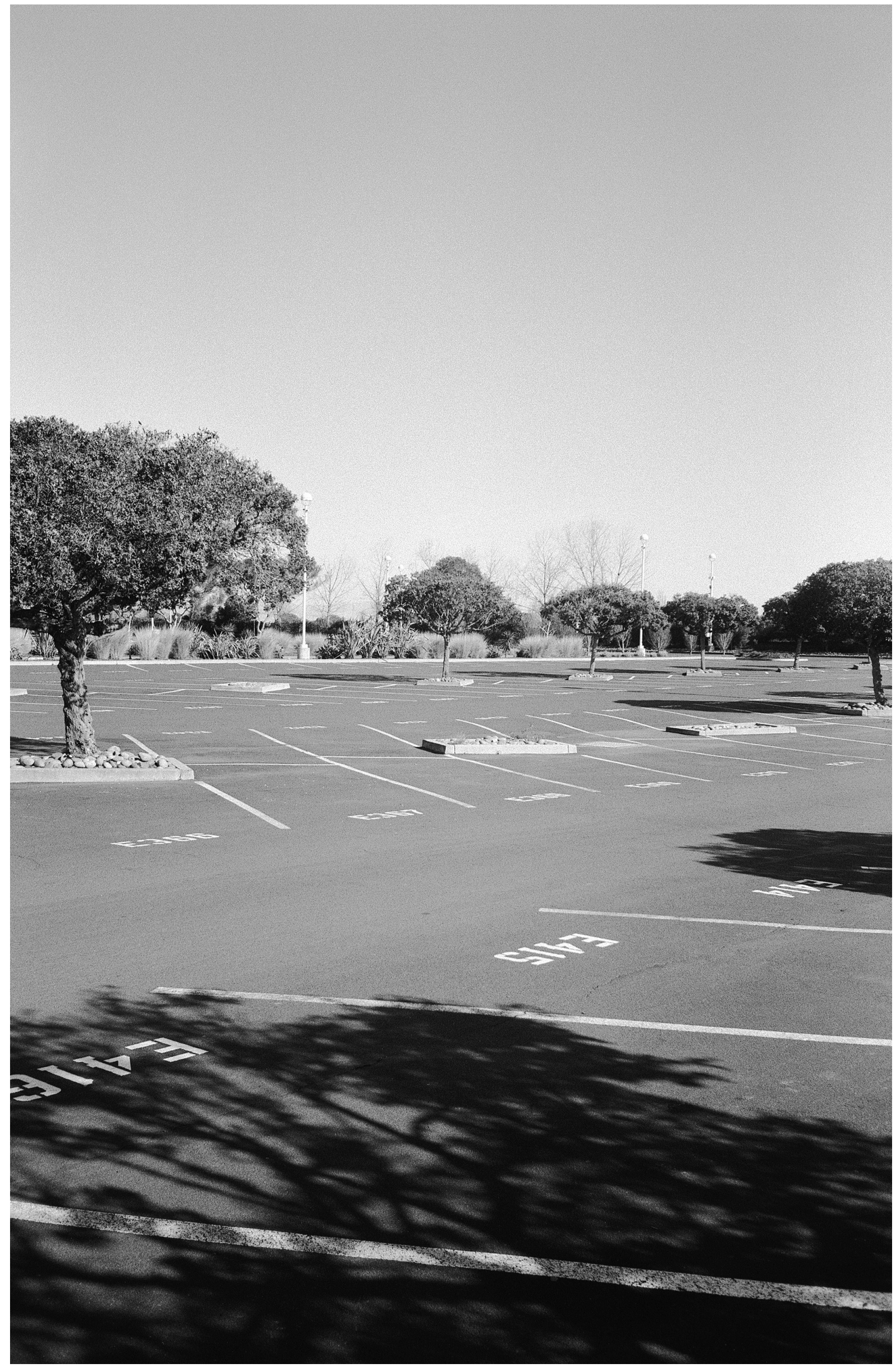




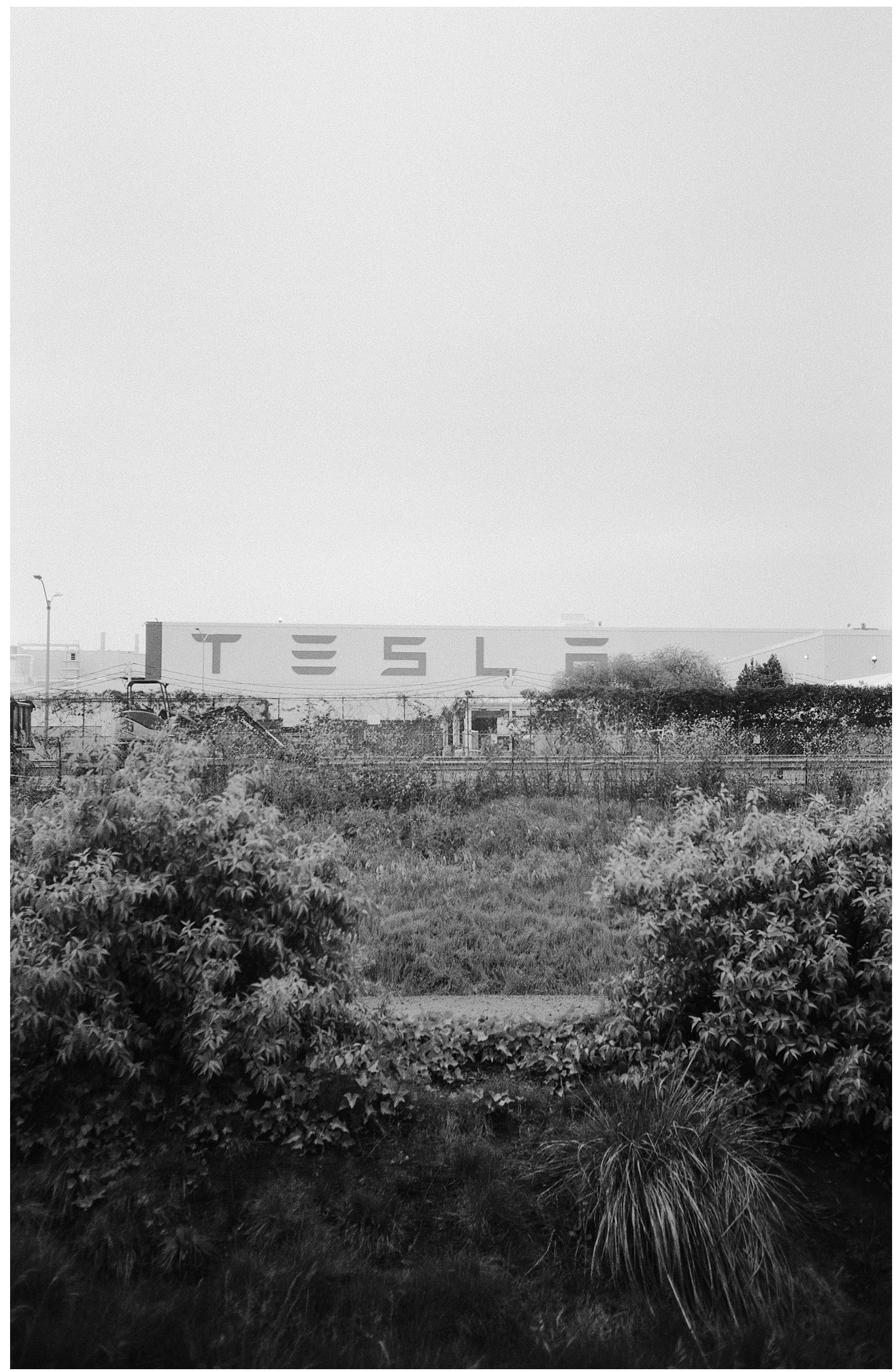




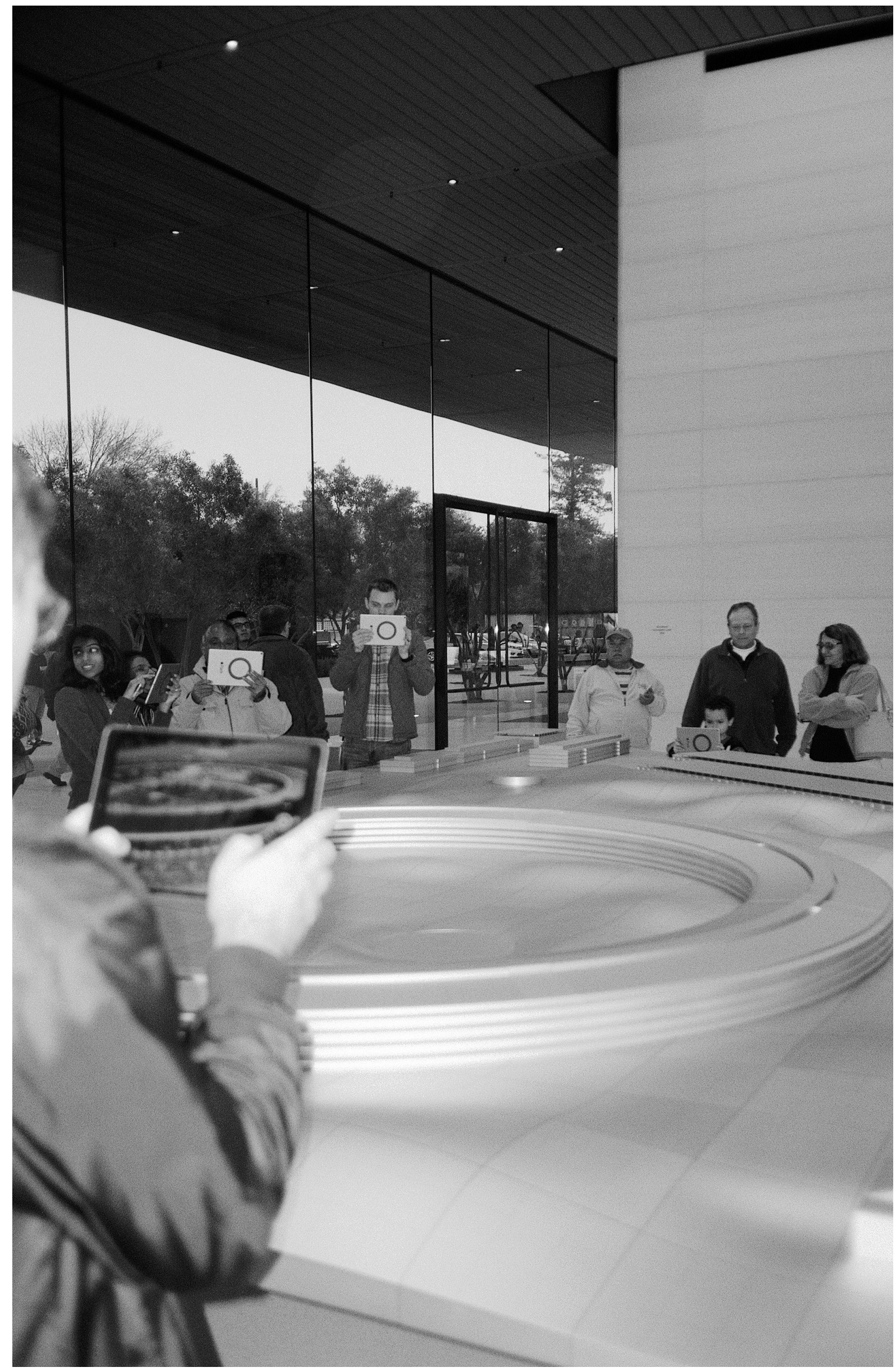




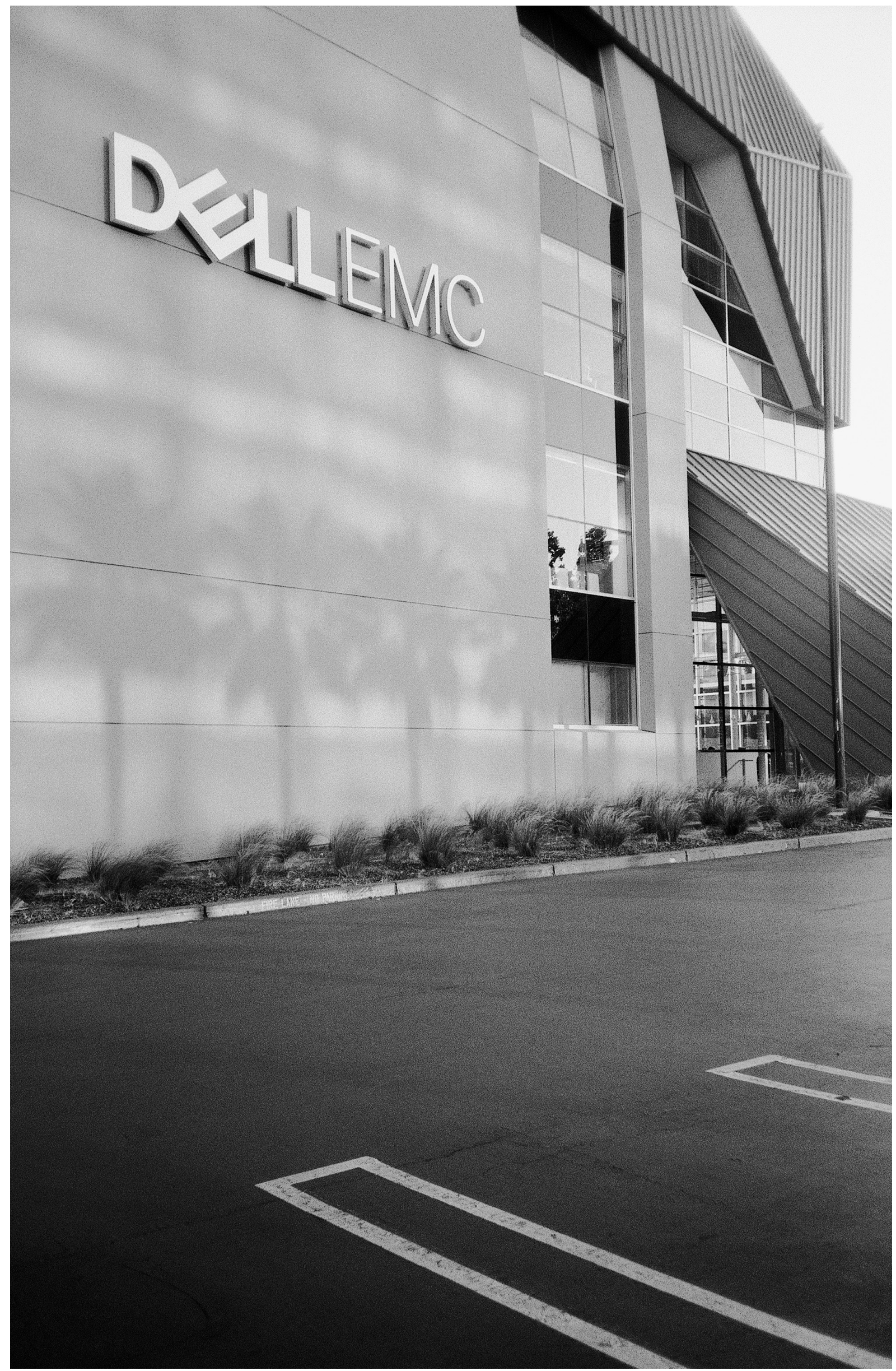




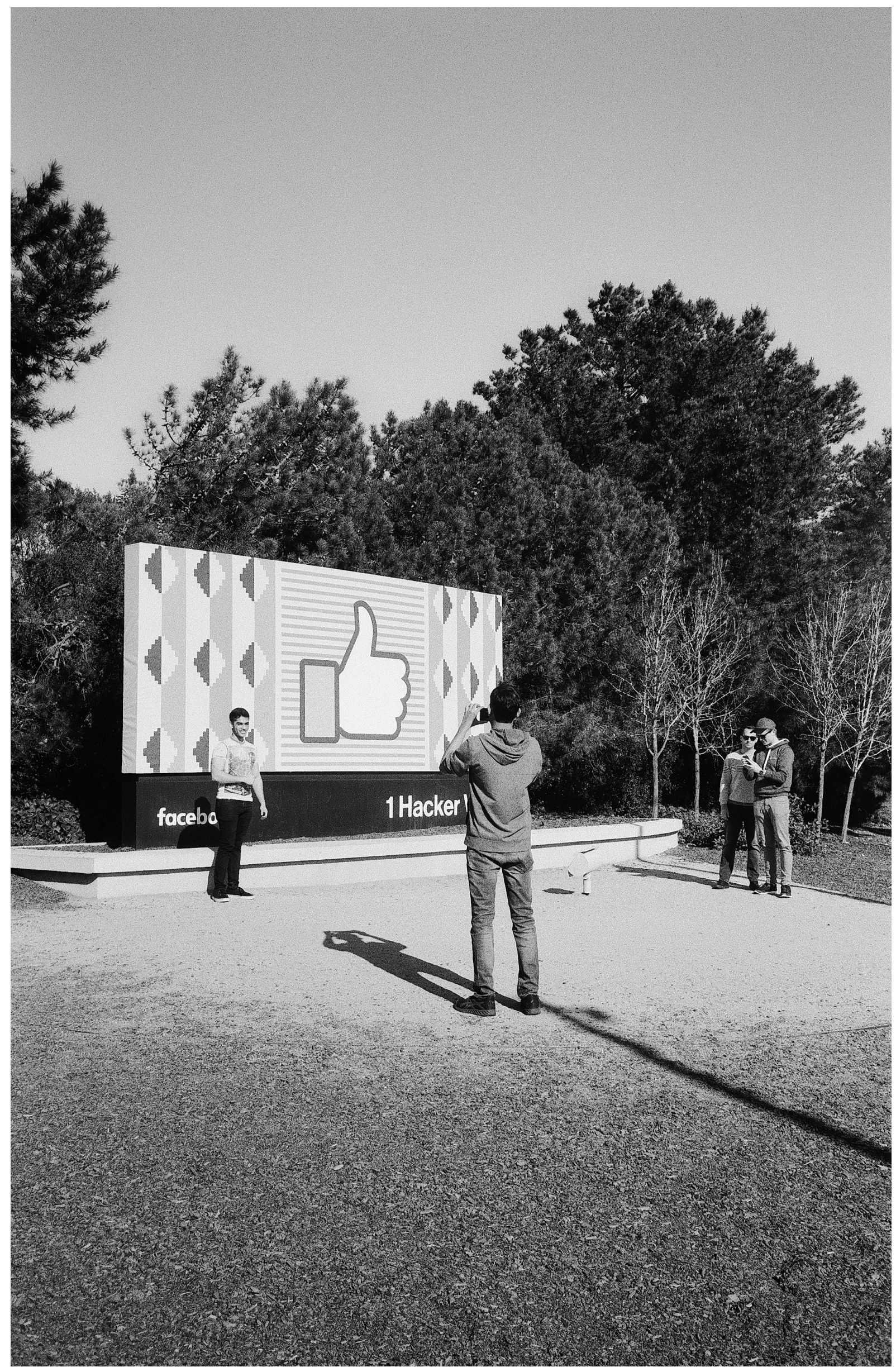




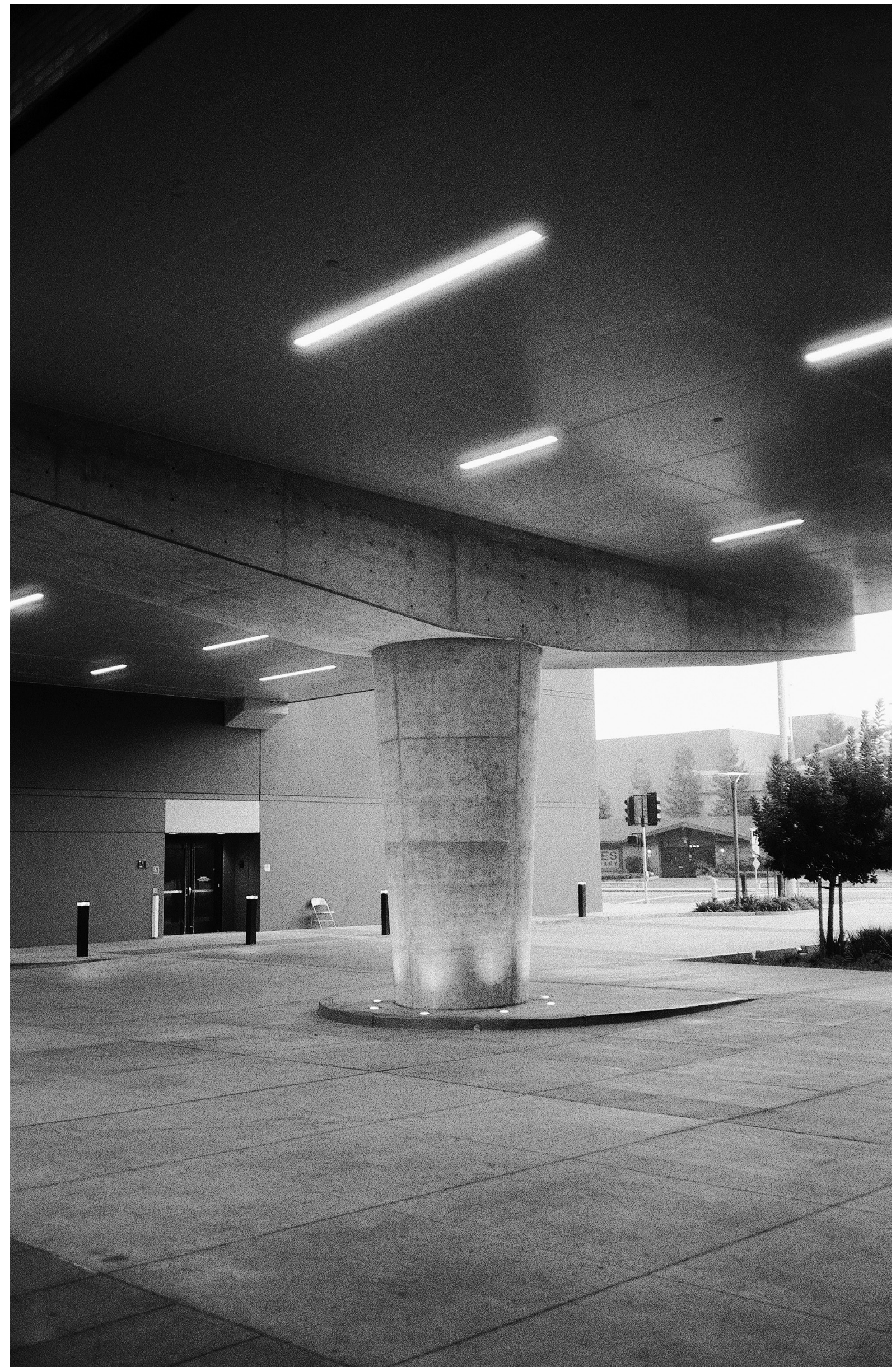




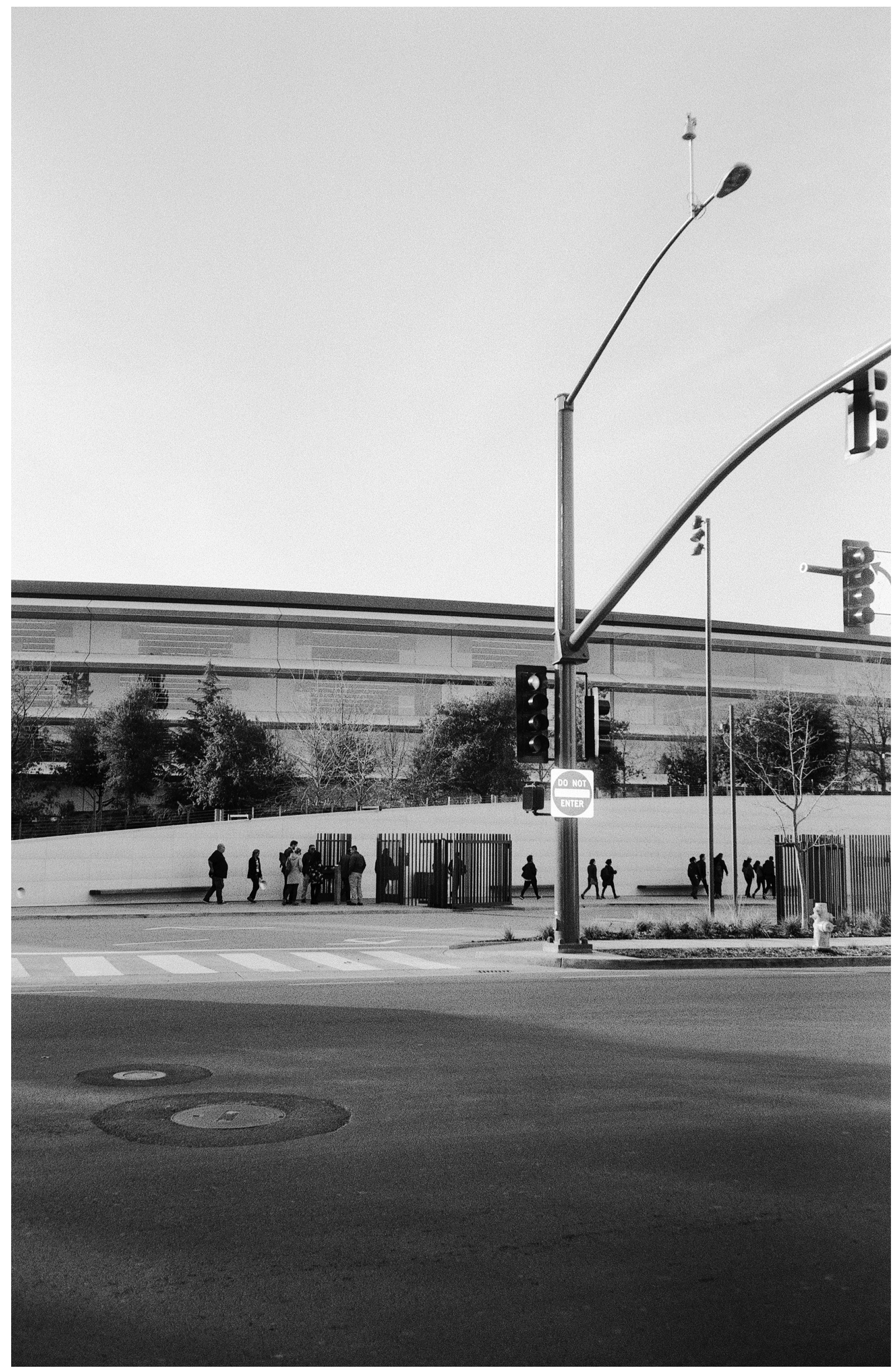




\section{Index}

1: Google Self Driving Car, Mountain View

2: Berkeley Lab, 1 Cyclotron Road, Berkeley

3: Here, 2075 Alston Ave, Berkeley

4: Facebook, 1 Hacker Way, Menlo Park

5: Tesla Factory, 4550o Fremont Boulevard, Fremont

6: Apple Park Visitor Center, 10600 N Tantau Ave, Cupertino

7: Dell Headquarters, 5455 Great America Park Way, Santa Clara

8: Facebook, 1 Hacker Way, Menlo Park

9: Amazon Webservice, 2100 University Ave, East Palo Alto

10: Apple Park, 10738 N Tantau Ave, Cupertino

\section{Kommentar}

In ihrem fotografischen Magazinbeitrag „Analog Algorithm - Landscapes of Machine Learning“, der auf ihrem gleichnamigen Buch basiert, nimmt uns die Fotografin Susanne Huth mit ins Silicon Valley, das Machtzentrum der technischen Innovation und der postindustriellen, neoliberalen Wirtschaftsordnung schlechthin. Mit ihren Arbeiten begibt sie sich auf einen Streifzug durch den etwa 70 Kilometer langen und 30 Kilometer breiten Landstrich in der San Francisco Bay Area, der Gegenstand globaler und kollektiver Fantasien zu sein scheint. Huths Bilder zeigen jedoch, dass es nicht ausreicht, lediglich über diesen Ort zu reden, zu behaupten, er habe mit seinen Technologien die Alltagsroutinen, Daten und digitale Identität einer jeden von uns erfasst und durchdrungen - ohne dass wir jemals da gewesen wären (Lübbke-Tidow 2020). Susanne Huths Schwarz-Weiß-Arbeiten brechen mit diesem Narrativ, indem sie das Silicon Valley als sozio-politischen Prozess beforschen, ihn als kulturelles Dispositiv begreifen und die strukturelle Transformation der Dienstleistungsgesellschaft zur Informationsgesellschaft nachzeichnen. Die dokumentierten Lagerhallen, Straßenzüge, Parkplätze, Gebäudekomplexe und Werbeplakate wirken nicht so schillernd oder innovativ wie die Marketingkampagnen der dort ansässigen Unternehmen, sondern brüchig und unspektakulär, fast schon alltäglich.

Bezugnehmend auf die New Topographics, eine Fotografie-Bewegung, die ihren Ursprung in den 1960er-Jahren im Westen der USA hatte, muten ihre Bilder als Landschaften an, die den Gründungsmythos der Vereinigten Staaten unterlaufen. Dieser begreift die vom Menschen veränderte Landschaft als Fundament der nationalen Imagination sowie als Legitimationsgrundlage für die Pionier_innenbewegung und die Unterdrückung von Native Americans, deren „Naturraum“ durch weiße Siedler_innen immer weiter in den Westen verdrängt wurde. Die Fotografin spürt diesen in die US-amerikanische Landschaft eingeschriebenen Machtasymmetrien im heutigen Silicon Valley nach. Dadurch gelingt es ihr, die sozialen Bedingungen der dortigen Kommunen, Forschungseinrichtungen und Unternehmen subtil herauszuschälen und so die großen Erzählungen von und über Big Tech und den Mythos des Digitalen zu durchkreuzen. 


\section{Autor_innen}

Susanne Huth ist Fotografin und Medienkünstlerin. Sie benutzt Fotografie, cross-media und Video-Installationen für ihre künstlerischen Forschungen im Feld der zeitgenössischen Stadtentwicklung.

post@susannehuth.de

\section{Literatur}

Huth, Susanne (2020): Analog Algorithm - Landscapes of Machine Learning. Salzburg: Fotohof edition.

Lübbke-Tidow, Maren (2020): Training Data. Zu Susanne Huths Analog Algorithm Landscapes of Machine Learning. In: Susanne Huth. Analog Algorithm - Landscapes of Machine Learning. Salzburg: Fotohof edition. 